\title{
Comparação da funcionalidade de idosos residentes em duas modalidades institucionais
}

Valéria Pagotto ${ }^{1}$, Valéria Alves Pereira da Silva ${ }^{2}$, Lilían Varanda Pereira ${ }^{3}$, Denise Pinheiro Marques Alves dos Santos ${ }^{4}$

\footnotetext{
${ }^{1}$ Enfermeira, Doutora em Ciências da Saúde. Professora Adjunta da Faculdade de Enfermagem da Universidade Federal de Goiás (FEN/UFG). Goiânia, GO, Brasil. Email: valeriapagotto@gmail.com.

${ }^{2}$ Discente do curso de Graduação em Enfermagem da FEN/UFG. Goiânia, GO, Brasil. E-mail: valeria.alves28@gmail.com.

${ }^{3}$ Enfermeira, Doutora em Enfermagem Fundamental. Professora Adjunta da FEN/UFG. Goiânia, GO, Brasil. E-mail: lilianvaranda7@gmail.com.

${ }^{4}$ Enfermeira, Mestre em Enfermagem. Docente da Universidade Paulista. Goiânia, GO, Brasil. E-mail:

enfermeiradenise@live.com.
}

Recebido: 28/03/2015.

Aceito: 24/09/2015.

Publicado: 31/03/2016.

Como citar esse artigo:

Pagotto V, Silva VAP, Pereira LV, Santos DPMA. Comparação da funcionalidade de idosos residentes em duas modalidades institucionais. Rev. Eletr. Enf. [Internet]. 2016 [acesso em:______ ];18:e1143. Disponível em: http://dx.doi.org/10.5216/ree.v18.34712.

\begin{abstract}
RESUMO
Objetivou-se comparar a prevalência e os fatores associados à incapacidade funcional para ABVD em idosos residentes em duas modalidades institucionais. Estudo transversal com 159 idosos de instituições de longa permanência, sendo 84 residentes em Casas Lares e 75 em Abrigos de Permanência Integral. A incapacidade funcional (IF) foi avaliada pelo Índice de Katz. As associações foram investigadas pelo Teste do $\chi^{2}$ ou Exato de Fischer. A prevalência de IF nas Casas Lares foi 16,7\% (IC95\%:8,0-28,8) e nos Abrigos de Permanência Integral 56,0\% (IC95\%44,2-68). Nas duas instituições prevaleceram hipertensão arterial e catarata. Nas Casas Lares, observou-se associação entre IF e idade $>80$ anos e relato de doenças osteomusculares. A depressão foi estatisticamente significante em ambas instituições. As ABVD mais comprometidas foram manter continência, vestir-se e banhar-se. Os achados demonstram a necessidade de intervenções junto aos idosos institucionalizados, principalmente moradores em instituições de modalidade permanência integral, com foco no controle da depressão.

Descritores: Idoso Fragilizado; Saúde do Idoso Institucionalizado; Enfermagem Geriátrica.
\end{abstract}

\section{INTRODUÇÃO}

A incapacidade funcional é definida como a dificuldade para realizar atividades da vida cotidiana e tarefas complexas de forma independente, além daquelas desejadas na sociedade ${ }^{(1)}$. Na avaliação clínica, ela é determinada por meio da capacidade do idoso para executar atividades da vida diária (AVD), seja por meio 
de atividades básicas (ABVD) ou instrumentais de vida diária (AIVD). As ABVD incluem atividades como alimentar-se, tomar banho, ir ao banheiro, manter a continência (urinária e fecal) e transferir-se $\mathrm{e}^{(2-3)}$.

Sabe-se que a perda ou diminuição da capacidade funcional resulta em perda da autonomia desses indivíduos para gerenciar a própria vida, comprometendo sua qualidade de vida ${ }^{(4)}$. Estudos demonstram que a incapacidade funcional em idosos da comunidade está associada ao sexo feminino, a baixa escolaridade, viuvez, baixa renda ${ }^{(3,5)}$, multimorbidade (três ou mais comorbidades) ${ }^{(6)}$. Além disso, a incapacidade funcional é forte preditora de mortalidade em idosos ${ }^{(3)}$.

No Brasil, estudos desenvolvidos com idosos institucionalizados nas regiões Sul, Sudeste e Nordeste ${ }^{(1,3,7-9)}$ demonstraram que a prevalência de incapacidade funcional varia de $10,3 \%$ a $70 \%$. Destacase que a população alvo desses estudos foram idosos residentes na modalidade institucional Abrigos de Permanência Integral e que a prevalência encontrada neles possui ampla variação, o que pode estar relacionada às disparidades sociais, econômicas e culturais entre as regiões brasileiras estudadas.

Com a crescente progressão do número de idosos na população mundial há um aumento na incidência de doenças incapacitantes e crônicas que podem comprometer a funcionalidade do idoso ${ }^{(10)}$ favorecendo sua institucionalização. No Brasil, existem diferentes modalidades de institucionalização do idoso, desde aquelas em que o idoso permanece de forma integral (Abrigos de Permanência Integral) e outras em que os idosos possuem maior autonomia para o cuidado (Casas Lar) ${ }^{(11)}$. Entretanto, pouco se sabe sobre as características da funcionalidade dos idosos nessas outras modalidades institucionais, tendo em vista que os estudos encontrados foram realizados com idosos em permanência integral ${ }^{(1,7-9)}$. Na prática profissional, observa-se que o tipo de institucionalização do idoso pode contribuir de forma diferente para o comprometimento nas AVD.

Sendo assim, este estudo traz importantes contribuições para o escopo de conhecimento das condições de saúde deste grupo e desvela informações sobre o nível de funcionalidade de idosos em modalidade de institucionalização (Casas Lar), e ainda agrega conhecimento sobre a funcionalidade de idosos em outra região brasileira, o que contribui para fomentar políticas públicas locais e nacionais.

Diante do exposto, o objetivo do estudo foi comparar a prevalência e os fatores associados à incapacidade funcional para ABVD em idosos residentes em duas modalidades institucionais.

\section{MATERIAIS E MÉTODOS}

Estudo transversal, cuja população alvo constituiu-se de pessoas que residiam em nove Instituições de Longa Permanência para Idosos (ILPI), cadastradas no município de uma capital do centro-oeste brasileiro, estimada em 346 pessoas, no ano de 2012. Destas ILPI, uma recusou-se a participar da pesquisa e uma foi excluída por abrigar passantes, moradores de rua.

Entre as sete ILPI participantes, quatro eram instituições tipo Casa Lar (CL) que se caracterizam pela residência individual e estímulo à realização de atividades de autocuidado, inclusive aquelas relacionadas às atividades básicas de vida diária. As outras três instituições eram do tipo Abrigo de Permanência Integral 
(API) que oferecem abrigo coletivo, sem espaços privativos para os residentes, atendidos em suas necessidades pela equipe assistencial, restritos ao ambiente de institucionalização e impossibilitados de se deslocarem da instituição sozinhos.

A inclusão dos idosos no estudo tomou como base o escore de função cognitiva avaliada por meio do Mini Exame do Estado Mental de igual ou superior a 13 (escore tomado pelos pesquisadores como limite para a execução das tarefas de medida solicitadas). Foram incluídos os residentes com 60 anos ou mais, sem restrições na fala, visão ou audição que impossibilitassem a coleta de dados, que residissem na ILPI (residir foi considerado como dormir quatro dias ou mais na instituição) e ser encontrado no momento da coleta de dados (foram feitas até três tentativas de encontro).

Após estudo piloto, os dados foram coletados no período de maio a agosto de 2012, por seis auxiliares de pesquisa previamente treinados para aplicação do questionário e dos instrumentos de medida. Para abordagem do idoso, inicialmente foi pré-agendado com o diretor das instituições o horário da coleta de dados. Os idosos foram entrevistados na maioria das vezes nos locais de convivência das instituições. Ao final da coleta de dados, realizou-se a checagem das informações e os dados foram digitados em dupla entrada para checagem de inconsistências.

A variável de desfecho foi a incapacidade funcional, avaliada por meio da escala de $\operatorname{Katz}^{(12)}$, que avalia a capacidade do idoso para executar atividades de vida diária (banho, vestir-se, ir ao banheiro, transferir-se, manter a continência, e alimentar-se) permitindo classificá-los em dependentes, parcialmente dependentes e independentes. A variável foi dicotomizada em dependentes (idosos dependentes e parcialmente dependentes) e independentes ${ }^{(6,13)}$.

Já as variáveis de exposição analisadas foram: demográficas (sexo, idade, estado civil, ter ou não filhos, escolaridade, tempo de institucionalização); doenças autorreferidas (diabetes, hipertensão arterial, hipercolesterolemia, osteoporose, câncer, catarata, acidente vascular cerebral, infarto agudo do miocárdio, asma ou bronquite, doenças osteomusculares); condições de saúde e clínicas (autoavaliação de saúde, multimorbidades, prática de atividade física, dor crônica e depressão). As doenças autorreferidas foram identificadas por meio da resposta à pergunta: "Quais destas doenças o médico já disse que o(a) Sr.(a) tem?”. As multimorbidades foram classificadas conforme o número de doenças autorreferidas, sendo aqueles que apresentavam cinco doenças ou mais. A autoavaliação de saúde foi mensurada por meio da resposta do idoso à pergunta: Em geral o(a) Sr.(a) diria que sua saúde é..", cujas opções de resposta eram: ótima, boa, regular, ruim ou péssima ${ }^{(14)}$. A depressão foi avaliada por meio da Escala de Depressão Geriátrica que é um dos instrumentos mais utilizados para rastreamento de depressão em idosos, e oferece medidas válidas e confiáveis $^{(15)}$.

As análises estatísticas foram realizadas no programa SPSS versão 20.0. Primeiramente todas as variáveis foram analisadas de forma descritiva: as variáveis categóricas foram expressas em frequências absolutas e relativas e as variáveis contínuas em médias e desvio-padrão. Estimou-se a prevalência de incapacidade funcional e a associação entre a incapacidade e as variáveis de exposição foi avaliada por meio 
do Teste qui-quadrado ou Teste Exato de Fischer, considerando um nível de significância de $5 \%(p<0,05)$.

O projeto foi aprovado pelo Comitê de Ética da Universidade Federal de Goiás-UFG, (Protocolo no398/11), respeitando legislação brasileira para pesquisa com seres humanos.

\section{RESULTADOS}

Dos 159 idosos que participaram da pesquisa, 52,9\% residiam em ILPI tipo Casa Lar e 47,1\% em ILPI tipo Abrigo de Permanência Integral. Nas Casas Lar prevaleceram idosos com idade entre 70 e 79 anos (51,2\%), sem companheiros (75\%), com filhos $(70,2 \%)$ e com ensino primário (53,6\%). Já nos Abrigos de Permanência Integral a maioria possuía 80 anos ou mais $(49,3 \%)$, sem companheiro $(70,7 \%)$ e com filhos (57,3\%). Em ambas modalidades houve predomínio de mulheres.

A prevalência de incapacidade funcional foi superior no do sexo masculino $(28,6 \%)$, de 70 a 79 anos (14\%), sem companheiro $(20,6 \%)$ e com tempo de institucionalização acima de cinco anos $(23,7 \%)$ nas Casas Lar. Nos Abrigos de Permanência Integral a prevalência foi superior no sexo feminino $(66,7 \%)$, nos muito idosos $\geq 80$ anos $(64,9 \%)$, sem companheiro $(61 \%)$ e com tempo de institucionalização entre um a cinco anos (60\%). Houve diferença estatisticamente significante entre incapacidade funcional e faixa etária de 80 anos ou mais $(p=0,048)$ nos Abrigos de Permanência Integral (Tabela 1$)$.

Tabela 1: Distribuição dos idosos, prevalência de incapacidade funcional e associação com fatores sociodemográficos. Goiânia, GO, Brasil, 2012.

\begin{tabular}{|c|c|c|c|c|c|c|}
\hline \multirow{3}{*}{ Variáveis } & \multicolumn{3}{|c|}{ Casa lar } & \multicolumn{3}{|c|}{ Abrigo de permanência integral } \\
\hline & \multirow{2}{*}{ Amostra $(n=84)$} & \multicolumn{2}{|c|}{ Prevalência incapacidade } & \multirow{2}{*}{ Amostra $(n=75)$} & \multicolumn{2}{|c|}{ Prevalência incapacidade } \\
\hline & & n (\%) & $p$ & & $\mathrm{n}(\%)$ & $p$ \\
\hline \multicolumn{7}{|l|}{ Sexo } \\
\hline Feminino & $49(58,3)$ & $4(8,7)$ & \multirow{2}{*}{0,276} & $36(48,0)$ & $24(66,7)$ & \multirow{2}{*}{0,074} \\
\hline Masculino & $35(41,7)$ & $10(28,6)$ & & $39(52,0)$ & $18(46,2)$ & \\
\hline \multicolumn{7}{|l|}{ Idade } \\
\hline $60-69$ anos & $15(17,9)$ & $1(6,7)$ & \multirow{3}{*}{0,077} & $15(20,0)$ & $5(33,3)$ & \multirow{3}{*}{$0,048^{*}$} \\
\hline 70-79 anos & $43(51,2)$ & $6(14,0)$ & & $23(30,7)$ & $13(56,5)$ & \\
\hline 80 anos ou mais & $26(31,0)$ & $7(26,9)$ & & $37(49,3)$ & $24(64,9)$ & \\
\hline \multicolumn{7}{|l|}{ Estado Civil } \\
\hline Com companheiro & $21(25,0)$ & $1(4,8)$ & \multirow{2}{*}{$0,081^{+}$} & $15(20,3)$ & $5(33,3)$ & \multirow{2}{*}{0,054} \\
\hline Sem companheiro & $63(75,0)$ & $13(20,6)$ & & $59(79,7)$ & $36(61,0)$ & \\
\hline \multicolumn{7}{|l|}{ Tem filhos } \\
\hline Sim & $59(70,2)$ & $10(16,9)$ & \multirow{2}{*}{$0,595^{\dagger}$} & $43(57,3)$ & $26(60,5)$ & \multirow{2}{*}{0,367} \\
\hline Não & $25(29,8)$ & $4(16,0)$ & & $32(42,7)$ & $16(50,0)$ & \\
\hline \multicolumn{7}{|l|}{ Escolaridade } \\
\hline Analfabeto & $20(23,8)$ & $4(20,0)$ & \multirow{5}{*}{0,891} & $18(24,3)$ & $9(50,0)$ & \multirow{5}{*}{0,167} \\
\hline Sabe ler e escrever & $10(11,9)$ & $2(20,0)$ & & $8(10,8)$ & $3(37,5)$ & \\
\hline Primário & $45(53,6)$ & $7(15,6)$ & & $35(47,3)$ & $20(57,1)$ & \\
\hline Ensino Médio & $5(6,0)$ & $1(20,0)$ & & $11(14,9)$ & $9(81,8)$ & \\
\hline Superior & $4(4,8)$ & $0(0,0)$ & & $2(2,7)$ & $1(50,0)$ & \\
\hline \multicolumn{7}{|c|}{ Tempo de institucionalização } \\
\hline$<1$ ano & $27(32,1)$ & $1(6,7)$ & \multirow{3}{*}{$0,104^{*}$} & $11(15,1)$ & $8(72,7)$ & \multirow{3}{*}{0,064} \\
\hline 1 a 5 anos & $18(21,4)$ & $4(12,9)$ & & $40(54,8)$ & $24(60,0)$ & \\
\hline$>5$ anos & $39(46,4)$ & $9(23,7)$ & & $22(30,1)$ & $9(40,9)$ & \\
\hline
\end{tabular}

*Qui-quadrado para tendência

** Teste exato de Fisher 
Na Tabela 2, observa-se que em ambas as modalidades, as doenças autorreferidas mais prevalentes na amostra geral foram hipertensão arterial e catarata. Entretanto, a prevalência de incapacidade funcional foi maior nos idosos com doenças osteomusculares em ambas modalidades; e em idosos com osteoporose e hipercolesterolemia (73,3\%) nos Abrigos de Permanência Integral. Porém, foram observadas associações estatisticamente significantes naqueles que relataram doenças osteomusculares ( $p=0,047)$ nas Casas Lar.

Tabela 2: Caracterização dos idosos institucionalizados com condições crônicas autorreferidas e associação à incapacidade funcional. Goiânia, GO, Brasil, 2012.

\begin{tabular}{|c|c|c|c|c|c|c|}
\hline \multirow{3}{*}{ Variáveis } & \multicolumn{3}{|c|}{ Casa lar } & \multicolumn{3}{|c|}{ Abrigos de permanência integral } \\
\hline & \multirow{2}{*}{ Amostra $(n=84)$} & \multicolumn{2}{|c|}{ Prevalência incapacidade } & \multirow{2}{*}{ Amostra $(n=75)$} & \multicolumn{2}{|c|}{ Prevalência incapacidade } \\
\hline & & n (\%) & $p$ & & n (\%) & $p$ \\
\hline Diabetes Mellitus & $17(20,2)$ & $4(23,5)$ & 0,395 & $13(17,3)$ & $7(53,8)$ & 0,863 \\
\hline Hipertensão Arterial & $45(53,6)$ & $7(15,6)$ & 0,769 & $39(52,0)$ & $22(56,4)$ & 0,941 \\
\hline Hipercolesterolemia & $30(35,7)$ & $3(10,0)$ & 0,222 & $15(20,3)$ & $11(73,3)$ & 0,147 \\
\hline Osteoporose & $28(33,3)$ & $7(25,0)$ & 0,147 & $15(20,0)$ & $11(73,3)$ & 0,131 \\
\hline Câncer & $5(6,0)$ & $1(20,0)$ & 0,837 & $5(6,8)$ & $1(20,0)$ & 0,086 \\
\hline Catarata & $49(58,3)$ & $11(22,4)$ & 0,092 & $33(44,0)$ & $18(54,5)$ & 0,822 \\
\hline AVC & $11(13,1)$ & $1(9,1)$ & 0,470 & $23(30,7)$ & $13(56,5)$ & 0,952 \\
\hline IAM & $5(6,0)$ & $0(0,0)$ & 0,302 & $5(6,7)$ & $1(20,0)$ & 0,093 \\
\hline Problemas respiratórios & $13(15,5)$ & $2(15,4)$ & 0,893 & $12(16,0)$ & $5(41,7)$ & 0,275 \\
\hline Doenças osteomusculares & $34(40,5)$ & $9(26,5)$ & 0,047 & $19(25,3)$ & $14(73,7)$ & 0,072 \\
\hline
\end{tabular}

Quanto às demais condições clínicas e de saúde, observa-se nas Casas Lar prevalência de incapacidade funcional superior nos idosos com autopercepção de saúde ruim (40\%), com mais de cinco doenças crônicas autorreferidas $(23,8 \%)$, que não realizavam nenhuma atividade física $(18,5 \%)$ e que apresentavam quadro de depressão (28,6\%). Nos Abrigos de Permanência Integral a prevalência de incapacidade funcional foi superior no grupo daqueles que referiram saúde como ruim $(72,2 \%)$, duas a cinco morbidades $(55,6 \%)$, dor crônica $(60,6 \%)$, e que apresentavam depressão $(65,9 \%)$. Observou-se diferenças estatisticamente significantes entre incapacidade funcional e depressão em ambas modalidades ( $p=0,042$ e $p=0,032$ ) (Tabela 3).

As ABVD com maior frequência de dependência foram manter continência, seguido de vestir-se e banhar-se em ambas instituições, sendo que nos Abrigos de Permanência Integral todas as ABVD apresentaram algum nível de comprometimento (Figura 1). 
Tabela 3: Caracterização dos idosos quanto a variáveis clínicas, e associação com a incapacidade funcional. Goiânia, GO, Brasil, 2012.

\begin{tabular}{|c|c|c|c|c|c|c|}
\hline \multirow{3}{*}{ Variáveis } & \multicolumn{3}{|c|}{ Casa lar } & \multicolumn{3}{|c|}{ Abrigo de permanência integral } \\
\hline & \multirow{2}{*}{ Amostra $(n=84)$} & \multicolumn{2}{|c|}{ Prevalência de incapacidade } & \multirow{2}{*}{ Amostra $(n=75)$} & \multicolumn{2}{|c|}{ Prevalência de incapacidade } \\
\hline & & n (\%) & $P$ & & n (\%) & $P$ \\
\hline \multicolumn{7}{|c|}{ Autoavaliação de saúde } \\
\hline Ótima/Boa & $38(45,2)$ & $8(21,1)$ & \multirow{3}{*}{0,808} & $30(40,0)$ & $18(60,0)$ & \multirow{3}{*}{0,612} \\
\hline Regular & $41(48,8)$ & $4(9,8)$ & & $27(36,0)$ & $11(40,7)$ & \\
\hline Ruim/Péssima & $5(6,0)$ & $2(40,0)$ & & $18(24,0)$ & $13(72,2)$ & \\
\hline \multicolumn{7}{|l|}{ Multimorbidade } \\
\hline 2 a 5 & $48(69,6)$ & $8(16,7)$ & \multirow{2}{*}{0,485} & $45(76,3)$ & $25(55,6)$ & \multirow{2}{*}{0,716} \\
\hline Mais de 5 & $21(30,4)$ & $5(23,8)$ & & $14(23,7)$ & $7(50,0)$ & \\
\hline \multicolumn{7}{|c|}{ Prática de atividade física } \\
\hline Sim & $57(67,9)$ & $9(15,8)$ & \multirow{2}{*}{0,754} & $25(33,3)$ & $14(56,0)$ & \multirow{2}{*}{1,000} \\
\hline Não & $27(31,2)$ & $5(18,5)$ & & $50(66,4)$ & $28(56,0)$ & \\
\hline \multicolumn{7}{|l|}{ Dor crônica } \\
\hline Sim & $45(53,6)$ & $8(17,8)$ & \multirow{2}{*}{0,769} & $33(44,0)$ & $20(60,6)$ & \multirow{2}{*}{0,476} \\
\hline Não & $39(46,4)$ & $6(15,4)$ & & $42(56,0)$ & $22(52,4)$ & \\
\hline \multicolumn{7}{|l|}{ Depressão } \\
\hline Sim & $28(33,7)$ & $8(28,6)$ & \multirow{2}{*}{0,042} & $41(56,2)$ & $27(65,9)$ & \multirow{2}{*}{0,032} \\
\hline Não & $55(66,3)$ & $6(10,9)$ & & $32(43,8)$ & $13(40,6)$ & \\
\hline
\end{tabular}

* Qui-quadrado para tendência

** Teste exato de Fisher

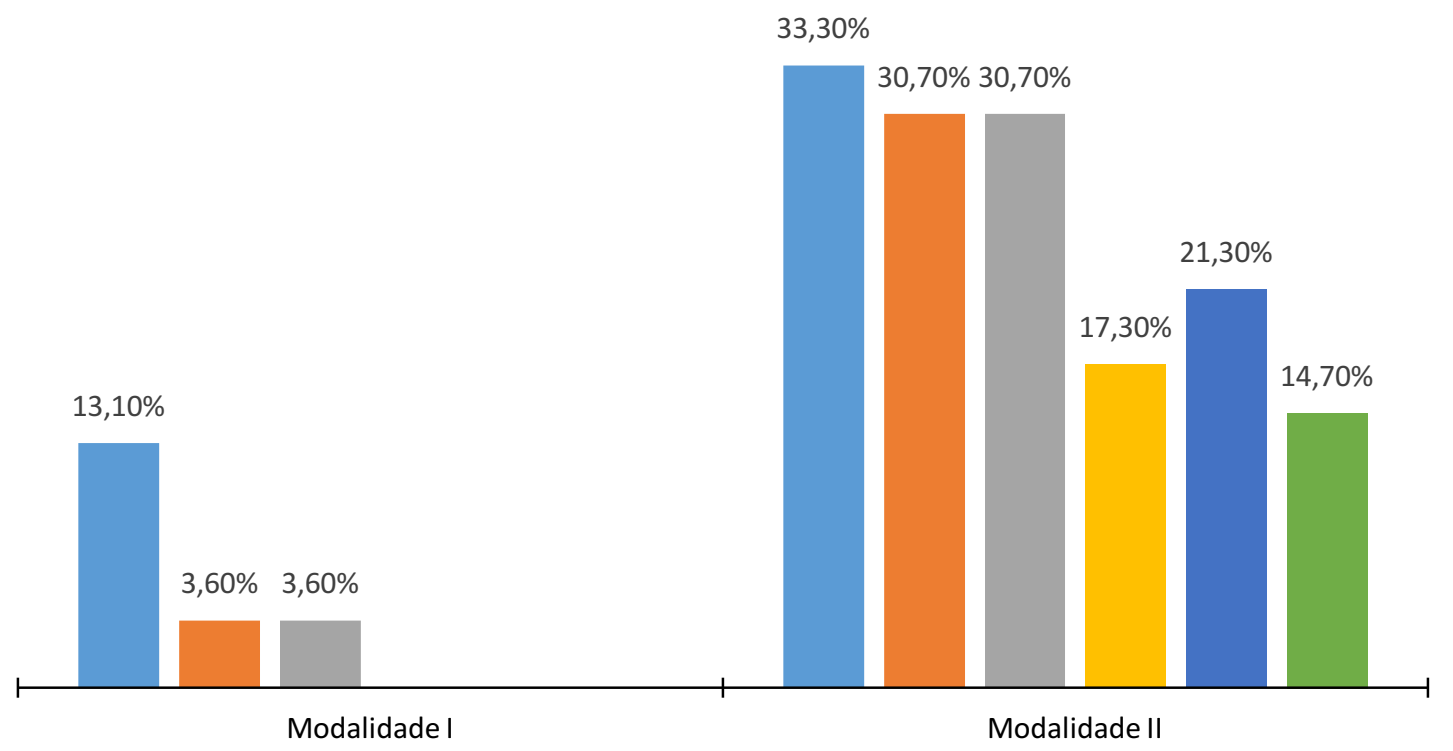

Figura 1: Frequência de ABVD com maior comprometimento. Goiânia, GO, Brasil, 2012.

\section{DISCUSSÃO}

A prevalência de incapacidade funcional foi superior nos idosos residentes em Abrigos de Permanência Integral comparando-se àqueles das Casas Lares, resultados esperados, tendo em vista que a capacidade para o autocuidado é requisito para inclusão do idoso nesta modalidade institucional. Apesar disso, a partir das análises, observou-se aumento da prevalência de incapacidade com o aumento do tempo de institucionalização, sugerindo que a incapacidade aumenta conforme aumenta o tempo de permanência do 
idoso nestas instituições. A prevalência encontrada neste estudo é inferior ao encontrado em estudos prévios desenvolvidos em Jequié $(B A)^{(1)}$, Montes Claros $(M G)^{(6)}$ e Pelotas $(R S)^{(3)}$, onde as prevalências de dependência para incapacidade funcional foram respectivamente 70\%, 56,8\% e 79,4\%. Em países da Europa, que já experimentam um envelhecimento populacional mais longo que a realidade brasileira, os resultados são similares. Na Espanha estudo conduzido com mais de 700 idosos institucionalizados, demonstrou que aproximadamente $80 \%$ apresentavam dependência para alguma $A B V D^{(16)}$. Na Finlândia, idosos que recebiam cuidados de longo prazo eram particularmente dependentes para as ABVD com limitações importantes para alimentação (aproximadamente $70 \%$ ) e necessidade de auxílio para banho $(99 \%)^{(17)}$. A menor prevalência de dependência encontrada neste estudo pode estar relacionada ao fato dos idosos participantes terem sido incluídos após obter a pontuação mínima no MiniMental $(\geq 13)$, de tal maneira que aqueles com déficit cognitivo severo e, presumivelmente, aqueles com maior nível de incapacidade funcional, não tenham sido alcançados, já que a função cognitiva está intimamente relacionada com o desempenho das atividades de vida diária ${ }^{(18)}$.

Comparando as duas instituições, observou-se que os idosos das Casas Lares apresentaram menor frequência de comprometimento nas ABVD. Entretanto, em ambas, as atividades mais comprometidas foram nesta ordem: manter continência, vestir-se e banhar-se. Nos Abrigos de Permanência Integral, os idosos ainda apresentam comprometimento para as demais ABVD. Essa diferença pode ser explicada pelo fato de que os indivíduos, para serem admitidos em Instituições de Longa Permanência com regime de Casa-lar devem apresentar maior independência, já que não serão assistidos em tempo integral como na outra modalidade discutida. Os estudos brasileiros com idosos institucionalizados demonstraram comprometimento em diferentes ABVD: transferir-se, tomar banho, vestir-se $\mathrm{e}$ ir ao banheiro ${ }^{(1,3-4)} \mathrm{e}$ deambular ${ }^{(1)}$. Existem evidências de que a perda da capacidade funcional inicia-se com o comprometimento das atividades instrumentais e posteriormente há o acometimento das ABVD tendo em vista que as instrumentais apresentam maior complexidade e são relacionadas à participação social do sujeito na sociedade ${ }^{(19-20)}$.

O nível de dependência também pode aumentar na medida em que os profissionais de saúde e/ou cuidadores dos idosos institucionalizados, muitas vezes optam por ajudá-los a executar determinadas tarefas básicas, ao invés de estimulá-los em suas dificuldades cotidianas ${ }^{(13)}$.

Em relação às características demográficas, observou-se maior frequência de incapacidade entre as mulheres nos Abrigos de Permanência Integral, e nos homens nas Casas Lares, embora não tenham apresentado diferenças estatisticamente significantes. Estudos prévios afirmam que a institucionalização ocorre mais entre as mulheres pelas diferenças entre sobrevivência e as morbidades associadas destacadas entre sexo feminino e masculino ${ }^{(3-4)}$. Além disso, sabe-se que embora as mulheres apresentem maior expectativa de vida que os homens, elas apresentam mais comorbidades, o que neste caso pode ter contribuído para maior prevalência de incapacidade funcional nos Abrigos de Permanência Integral. Ao contrário, como as Casas Lares caracterizam-se por admitir idosos independentes, as mulheres podem ter 
apresentado maior independência comparado aos homens porque assumem seus compromissos com os cuidados com a saúde.

Houve elevação na prevalência de incapacidade com o aumento da faixa etária, resultado que é

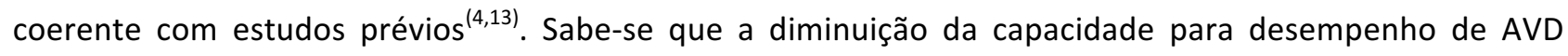
aumenta com a idade e culmina com a necessidade de cuidados a longo prazo, seja por familiares, amigos, vizinhos (informalmente), ou por profissionais capacitados ${ }^{(3)}$, o que pode levar à institucionalização do idoso.

Entre as comorbidades, a depressão apresentou diferenças estatisticamente significantes à incapacidade funcional nas duas modalidades. Os idosos institucionalizados tem pouco acesso a atividades de lazer e convivência já que essas instituições estão mais focadas no cuidado relacionado à higiene pessoal e auxílio nas atividades cotidianas, deixando de lado atividades de lazer e saúde. Além disso, o envelhecimento leva à diminuição da funcionalidade e a piora da qualidade de vida desse idoso, o que o deixa mais suscetível a sintomas depressivos ${ }^{(20)}$.

As doenças osteomusculares também apresentaram associação com incapacidade nas Casas Lar $(p=0,047)$. Como tratam-se de idosos mais ativos, a presença de doenças osteomusculares pode afetar o desempenho de atividades cotidianas, visto que podem limitá-los a executar as atividades fora daquele local. No caso dos Abrigos de Permanência Integral, a presença dessas doenças pode estar relacionada ao fato de esses idosos não estarem ativos funcionalmente, não realizarem atividades físicas, e ficarem restritos à instituição.

\section{CONCLUSÃO}

A prevalência de IF foi superior nos Abrigos de Permanência Integral em comparação as Casas Lar e a depressão esteve associada a incapacidade em ambas. As doenças osteomusculares nas Casas Lares e a faixa etária acima de 80 anos nos Abrigos de Permanência Integral também foram variáveis associadas a incapacidade funcional. As ABVD com maior comprometimento foram em ambas manter continência, banhar-se e vestir-se.

Apesar das contribuições, este estudo apresenta algumas limitações. Não foi analisada a totalidade dos idosos. Entretanto, isso garantiu a fidedignidade dos dados, uma vez que idosos com déficit cognitivo podem dar informações imprecisas e levar a vieses. Outra limitação refere-se ao fato de que estudos transversais não permitem distinguir causa de efeito, ou seja, não pode-se inferir causalidade entre as variáveis de exposição associadas e o desfecho, o que poderá ser estudado em estudos de coorte.

Os achados sinalizam a necessidade de intervenções junto aos idosos institucionalizados, principalmente moradores em instituições de modalidade permanência integral, com foco no controle da depressão.

O enfermeiro tem o papel fundamental nos cenários das Instituições de Longa Permanência. Frente aos achados recomenda-se que a atuação do enfermeiro seja potencializar o envelhecimento ativo em idosos residentes em instituições similares à Casas Lar, em que o idoso possui um nível de dependência menor e 
propor ações que previnam incapacidades entre aqueles que vivem em instituições do tipo permanência integral. Em ambas instituições, a avaliação do nível de dependência e a oferta de ações, cujo foco seja o desenvolvimento das funções motoras e cognitivas, são propostas que podem ser implementadas e avaliadas continuamente pelo profissional de enfermagem.

Recomenda-se que futuros estudos investiguem a funcionalidade de idosos em outras regiões do país e em outras modalidades institucionais, além das duas pesquisadas, para fomentar políticas cujo foco sejam os cuidados de longa duração do idoso, com qualidade na atenção e com foco na manutenção da autonomia e independência do idoso.

\section{REFERÊNCIAS}

1. Reis LA, Torres GV. Influência da dor crônica na capacidade funcional de idosos institucionalizados. Rev Bras Enferm [Internet]. 2011 [acesso em: 31 mar. 2016];64(2):274-80. Disponível em:

http://www.scielo.br/scielo.php?script=sci_arttext\&pid=S0034-71672011000200009\&lng=en\&nrm=iso\&tlng=pt.

2. Fonseca M das GUP, Firmo JOA, Loyola Filho Al, Uchôa E. Papel da autonomia na auto-avaliação da saúde do idoso. Rev Saude Publica [Internet]. 2010 [acesso em: 31 mar. 2016];44(1):159-65. Disponível em: http://dx.doi.org/10.1590/S0034-89102010000100017.

3. Del Duca GF, Silva MC, Silva SG, Nahas MV, Hallal PC. Incapacidade funcional em idosos institucionalizados Revista Brasileira de Atividade Física \& Saúde [Internet]. 2011 [acesso em: 31 mar. 2016];16(2):120-4. Disponível em: https://periodicos.ufpel.edu.br/ojs2/index.php/RBAFS/article/view/569.

4. Marinho LM, Vieira MA, Costa SM, Andrade JMO. Degree of dependence of elderly residents in geriatric long-term care facilities in Montes Claros, MG. Rev Gaucha Enferm [Internet]. 2013 [acesso em: 31 mar. 2016];34(1):104-10. Disponivel em: http://dx.doi.org/10.1590/S1983-14472013000100013.

5. Alves LC, Leite IC, Machado CJ. Factors associated with functional disability of elderly in Brazil: a multilevel analysis. Rev Saude Publica [Internet]. 2010 [acesso em: 31 mar. 2016];44(3):468-78. Disponível em:

http://dx.doi.org/10.1590/S0034-89102010005000009.

6. Marengoni A, Angleman S, Melis R, Mangialasche F, Karp A, Garmen A, et al. Aging with multimorbidity: a systematic review of the literature. Ageing Res Rev [Internet]. 2011 [acesso em: 31 mar. 2016];10(4):430-9. Disponível em: http://dx.doi.org/10.1016/j.arr.2011.03.003.

7. Cardoso LS, Silva BT, Rodrigues DS, Leal CL, Penner MCS. Aged: functional capacity for basic and instrumental activities of daily living. Revista de Pesquisa: Cuidado é Fundamental Online [Internet]. 2014 [acesso em: 31 mar. 2016];6(2):584-93. Disponível em: http://dx.doi.org/10.9789/2175-5361.2014v6n2p584.

8. Smanioto FN, Haddad MCFL. Índice de Katz aplicado a idosos institucionalizados. Ver Rene [Internet]. 2011 [acesso em: 31 mar. 2016];12(1):18-23. Disponível em:

http://www.revistarene.ufc.br/revista/index.php/revista/article/view/686.

9. Sousa KT, Mesquita LAS, Pereira LA, Azeredo CM. Baixo peso e dependência funcional em idosos institucionalizados de Uberlândia (MG), Brasil. Cien Saude Colet [Internet]. 2014 [acesso em: 31 mar. 2016];19(8):3513-20. Disponível em: http://dx.doi.org/10.1590/1413-81232014198.21472013.

10. Andrade DMB, Nery VAS. Avaliação da qualidade de vida de idosos institucionalizados. Ciência \& Desenvolvimento - Revista Eletrônica da FAINOR [Internet]. 2012 [acesso em: 31 mar. 2016];5(1):130-40. Disponível em:

http://srv02.fainor.com.br/revista/index.php/memorias/article/view/163.

11. Araújo CLO, Souza LA, Faro ACM. Trajetória das instituições de longa permanência para idosos no Brasil. Hist enferm, Rev eletronica [Internet]. 2010 [acesso em: 31 mar. 2016];1(2):250-62. Disponível em:

http://www.here.abennacional.org.br/here/n2vol1ano1 artigo3.pdf.

12. Duarte $Y A O$, Andrade CL, Lebrão ML. O Índex de Katz na avaliação da funcionalidade dos idosos. Rev Esc Enferm USP [Internet]. 2007 [acesso em: 31 mar. 2016];41(2):317-25. Disponível em: http://dx.doi.org/10.1590/s008062342007000200021.

13. Barros JFP, Alves KCA de O, Filho AVD, Rodrigues JE, Neiva HC. Avaliação da capacidade funcional de idosos 
institucionalizados na cidade de Maceió - AL. Rev. bras. promoç. saúde (Impr.). [Internet]. 2012 [acesso em: 31 mar. 2016];23(3):168-74. Disponível em: http://dx.doi.org/10.5020/18061230.2010.p168.

14. Del Duca GF, Silva MC, Hallal PC. Disability in relation to basic and instrumental activities of daily living among elderly subjects. Rev Saude Publica [Internet]. 2009 [acesso em: 31 mar. 2016];43(5):796-805. Disponível em: http://dx.doi.org/10.1590/S0034-89102009005000057.

15. Paradela EMP, Lourenço RA, Veras RP. Validação da escala de depressão geriátrica em um ambulatório geral. Rev Saude Publica [Internet]. 2005 [acesso em: 31 mar. 2016];39(6):918-23. Disponível em:

http://dx.doi.org/10.1590/S0034-89102005000600008.

16. Damián J, Pastor-Barriuso R, Valderrama-Gama E, Pedro-Cuesta J. Discordance between physician-rated health and an objective health measure among institutionalized older people. BMC Geriatr [Internet]. 2015 [acesso em: 31 mar. 2016];15:78. Disponível em: http://dx.doi.org/10.1186/s12877-015-0074-4.

17. Laukkanen P, Karppi P, Heikkinen E, Kauppinen M. Coping with activities of daily living in different care settings. Age Ageing [Internet]. 2001 [acesso em: 31 mar. 2016];30(6):489-94. Disponível em:

http://dx.doi.org/10.1093/ageing/30.6.489.

18. De Vriendt P, Gorus E, Cornelis E, Bautmans I, Petrovic M, Mets T. The advanced activities of daily living: a tool allowing the evaluation of subtle functional decline in mild cognitive impairment. J Nutr Health Aging [Internet]. 2013 [acesso em: 31 mar. 2016];17(1):64-71. Disponível em: http://dx.doi.org/10.1007/s12603-012-0381-9.

19. Dias EG, Andrade FB, Duarte YAO, Santos JLF, Lebrão ML. Advanced activities of daily living and incidence of cognitive decline in the elderly: the SABE Study. Cad Saude Publica [Internet]. 2015 [acesso em: 31 mar.

2016];31(8):1623-35. Disponível em: http://dx.doi.org/10.1590/0102-311X00125014.

20. Silva ER, Sousa ARP, Ferreira LB, Peixoto HM. Prevalência e fatores associados à depressão entre idosos institucionalizados: subsídio ao cuidado de enfermagem. Rev Esc Enferm USP [Internet]. 2012 [acesso em: 31 mar. 2016];46(6):1387-93. Disponível em: http://dx.doi.org/10.1590/S0080-62342012000600015. 